\title{
Multi-Rate Segmented Time-Interleaved Current Steering DAC with Unity-Elements Sharing
}

\author{
Devrim Aksin ${ }^{(1)}$, Gurer Ozbek ${ }^{(1)}$ and Franco Maloberti ${ }^{(2)}$ \\ (1) Istanbul Technical University, Turkey (2) University of Pavia, Italy \\ E-mail: devrimaksin@ieee.org
}

\begin{abstract}
A novel multi-rate Time-Interleaved Current Steering Digital to Analog Converter with unity element sharing is presented. Proposed 12-bit DAC is simulated in 90nm CMOS technology. The implemented DAC is divided to two segments, MSB and LSB segments, each having 6 bits of resolution. Taking advantage of the oversampling requirement due to the reconstruction filter at the DAC output, the MSB segment is further separated to 6 subDACs to implement time-interleaved and return-to-zero techniques. Time interleaved technique reduces the switching speed of each individual unit element to $1 / 6^{t h}$ of the sampling clock frequency and RTZ technique improves the linearity of the DAC. Simulations show that employed technique improves the linearity over 6dB with an SFDR of $85 \mathrm{~dB}$ with 6GS/s sampling frequency and $460 \mathrm{MHz}$ input frequency. The DAC draws $13 \mathrm{~mA}$ from a $1.5 \mathrm{~V}$ supply. The expected active area of the DAC is $1 \mathrm{~mm}^{2}$.
\end{abstract}

\section{INTRODUCTION}

Modern communication systems, such as cellular base stations, require medium-high resolution DACs with large signal bands. Since the reconstruction filter needs a proper frequency margin, the conversion speed can be several times of the signal band and, for advanced architectures, become as high as many GS/s [1]. The suitable scheme for very high speed DACs is the current steering that uses unity current generators controlled according to a segmented code that provides the unary and the binary control. The use of thin line-width technology enable a very precise current switching with frequencies up to 1-2 $\mathrm{GHz}$ but obtaining higher speeds is difficult especially when the return-to-zero (RTZ) is recommended. With an $M+N=n$ segmentation the accuracy of the conversion of the $M$ MSB must be $2^{N}$ higher than the one of the LSB. Therefore, the architecture can foresee methods with different precision for the MSB DAC and for the LSB DAC.

The time-interleaved approach is normally used for ADCs. The clock of a single converter of the scheme is reduced because it works in an interleaved fashion, by processing decimated analog samples. This paper proposes to use the time-interleaved method for the MSB DAC. As will be clear shortly, the clock of a MSB DAC unit cell is lower than the conversion speed because it operates in an interleaved fashion. Moreover, the generated analog signal from each MSB unit cell lasts for a number of clock periods and provides RTZ. This specific architecture uses 6 current steering unit cell groups that deliver their currents for 4 clock periods. The unity elements of the MSB DAC are not specific to a DAC group but come from a single large set of elements capable to ensure

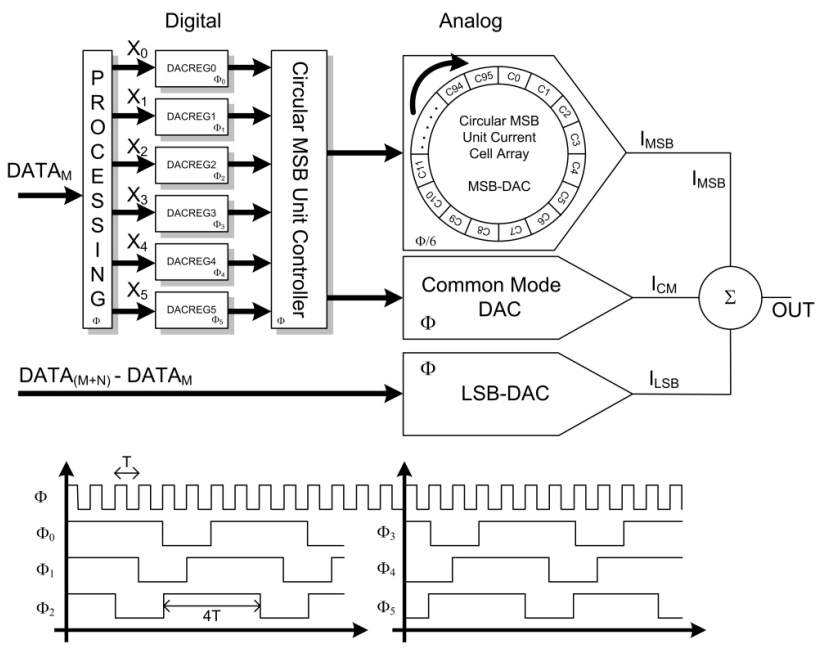

Fig. 1. The architecture of the proposed time-interleaved DAC

RTZ for at least two clock periods [2], [3]. The LSBs are converted with a full speed DAC. The following description of the method shows that the architecture with a $90 \mathrm{~nm}$ CMOS technology works properly at $6 \mathrm{GS} / \mathrm{s}$ and 12 bit of resolution.

\section{Time-INTERLEAVED ARCHiteCtURE}

Fig. 1 shows the proposed time-interleaved (TI) DAC. The LSB bits are converted by LSB DAC at full clock speed. The common mode correction DAC whose function will be explained later also operates at full speed.

The scheme utilizes 6 subDACs sharing a unified unit element array to generate MSB, 4 for TI and 2 for RTZ. However, the number of path of the TI and the RTZ can be generalized to any number. Each subDAC output is controlled by the respective DAC register. The output of each DAC operates under the control of the phases shown in the figure. Each output lasts for 4 clock periods and returns to zero for two clock periods. Actually, as will be clarified below, the implementation obtains a variable RTZ period, that is at least two clock periods. The MSB unit controller determine which section of the MSB unit cell array will be activated or deactivated. MSB current array contains only 96 bipolar unit current cells.

Since the outputs of the DACs are current, their addition is straightforward. The processing of the input data must be, obviously, such that for each clock period the output current 
gives the conversion of input. Therefore, at the time $N$, when DAC1 turns on, the current $I_{M S B}(N)$ must be the D/A conversion of the input $X(N)$. The activated unit cells are determined so that the output current at instant $N$ is

$$
\begin{gathered}
I_{M S B}(N)=\sum_{i=0}^{3} I_{D A C R E G, i}(N) \\
I_{D A C R E G 4}(N)=I_{D A C R E G 5}(N)=0
\end{gathered}
$$

where $I_{D A C R E G i}(N)$ is the current associated with the DAC register $i, i=0 . .5$. The next clock period $(N+1)$, one subDAC turns off and another turns on

$$
\begin{gathered}
X_{i}(N+1)=X_{i}(N) \text { for } i=0 . .2 \\
X_{3}(N+1)=X_{4}(N+1)=0
\end{gathered}
$$

with $X_{5}(N+1)$ to be determined. It must account for the change of the digital input and $X_{3}(N)$ that turns off. Therefore

$$
X_{5}(N+1)=X(N+1)-X(N)+X_{3}(N)
$$

In the next clock period, $X_{2}$ turns off and $X_{4}$ turns on, and so on. Therefore, the value of the turned on digital controls are calculated with the generalization of (5). The operation described above gives rise to a digital result that, thanks to the slight oversampling, is well within the full scale range. Therefore, the dynamic range of the parallel DACs can be limited to a fraction of the full range. The digital results are stored within the DAC registers.

\section{Simulations of the IdeAl Scheme}

The architecture of Fig. 1 has been simulated at the behavioral level with $M=N=6$ to convert a full scale 12bit digital input sine wave with frequency $1 / 13.85$ times the clock, corresponding to about 6.9 oversampling.

With the range of the MSB-DACs limited to half full range, the digital controls are the ones shown in Fig. 2. Notice that the sum of the DAC register at each time instant is equal to the input signal at the respective time instant. To show the RTZ behavior of DACREG0 that is a representative of DACREGs, DACREG0 signal together with the register sum signal is also provided within the figure.

The generated signals are bipolar and bounded to the fraction of the full scale range thanks to the oversampling. It is possible to implement the scheme by connecting directly the DAC register outputs to 6 separate 6-bit DACs. But this technique would require far more resources than actually required. The simulation results show that actually using 96 unit elements and sharing it in circular fashion among the DAC registers is enough to generate desired output under the oversampling condition.

The simulation also provides the total number of current sources used every clock period. This can be obtained by summing the absolute values of the individual DAC registers. The required number of bipolar unit cells at each time instant is shown in Fig. 3. At each time instant, required number of
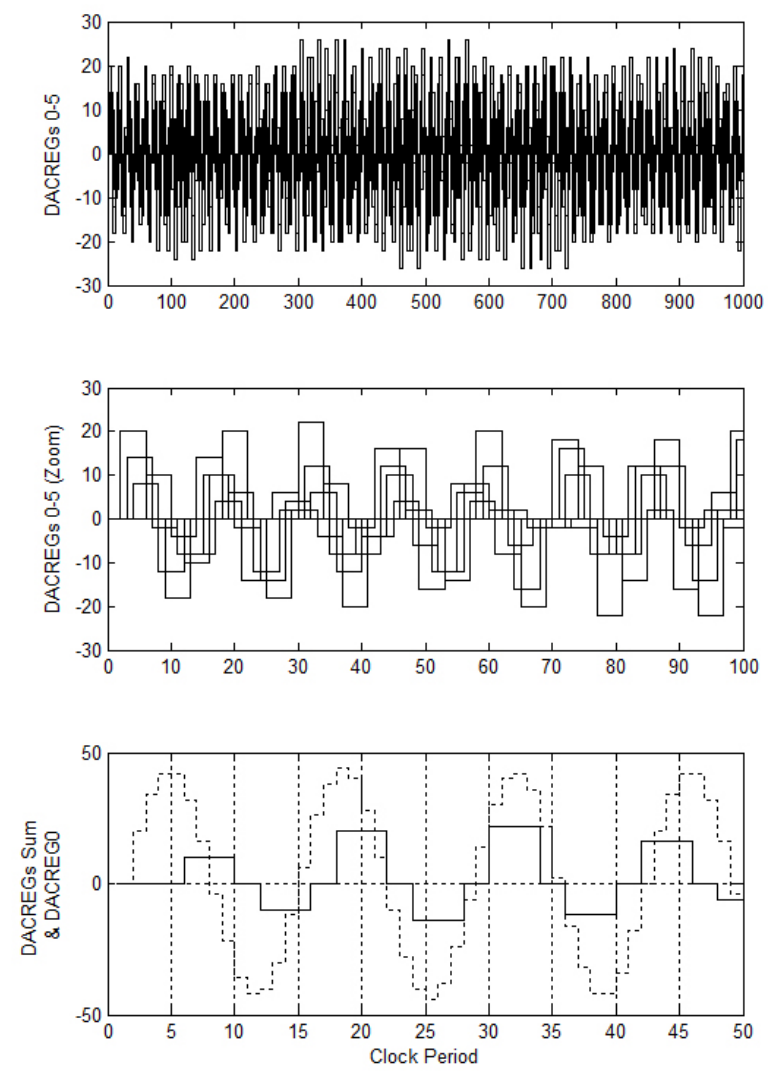

Fig. 2. The digital signals stored within the DACREGs

cells is bounded with the magnitude of the non zero subDAC output and as it is clear from the figure, the requirement is less than 64. Since the algorithm assumes at least 2 clock periods of zero output for each subDAC, it is necessary to include extra 32 unit cells to the MSB array.

In order to generate bipolar output, it is necessary to use differential output architecture. The output signals at the positive and negative terminals are shown in Fig. 4. As it is clear from the figure, although the outputs are generated in differential fashion, the output common mode voltage changes from sample to sample if a common mode correction is not present. The CM subDAC regulates the common voltage variation. The input code of the CM subDAC is calculated digitally at each clock period as follows:

$$
D A T A_{C M_{-} D A C}=C M-0.5\left(D A T A_{P}+D A T A_{N}\right)
$$

where $C M$ is the desired common mode level at the output, $D A T A_{P}$ and $D A T A_{N}$ are the number of active current cells connected to positive and negative outputs respectively. $D A T A_{P}$ and $D A T A_{N}$ values are available beforehand at each clock period for calculations. Note that the output common mode variation is actually equal to the half of the total number of active unit cells at each clock period, in other words it is half of the waveform given in Fig. 3. 


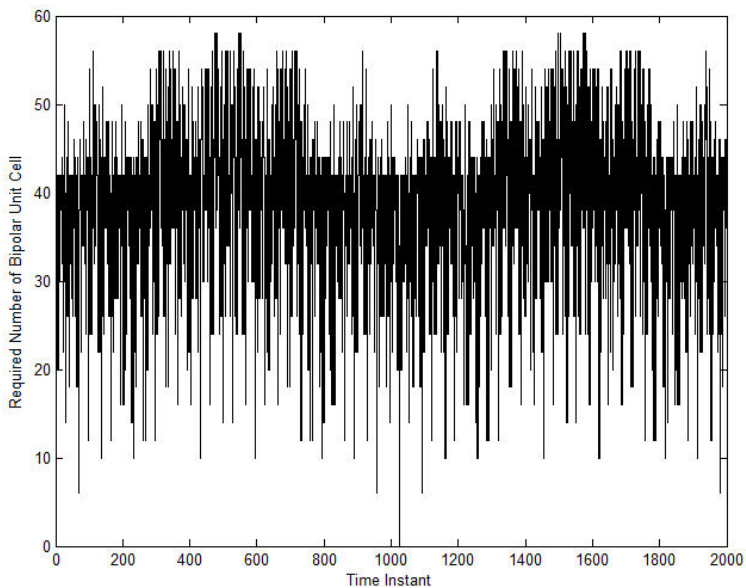

Fig. 3. Required number of bipolar unit cells

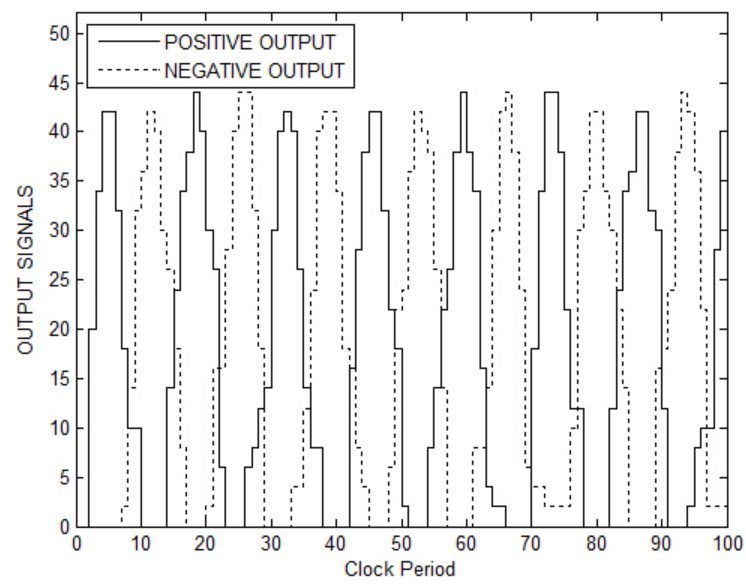

Fig. 4. The output signals at the positive and negative terminals

\section{UNITY ELEMENT SHARING}

As mentioned earlier, the simulations show that only 96 unit elements are necessary to implement the MSB section of 12-bit DAC. The circular MSB unit controller implement unit element sharing among the 6 subDACs whose values retained by the subDAC registers. MSB unit controller keeps track of two pointers, namely start pointer and stop pointer. The start pointer points the first active element and the stop pointer points the last one. In other words, only the unit elements in between the start and stop pointers are active and the remaining unit elements are all zero. When a pointer exceed the maximum available unit element, it restart cycling from beginning. Naturally, for the case at which the stop pointer is actually smaller than the start pointer, the unit elements between the pointers are made inactive and the unit elements outside of the pointers are active. This circular activation operation is obtained. This technique is very similar to the dynamic element matching employed within delta-sigma modulators.

At each time instant, the start pointer advances with the magnitude of the turned off subDAC and stop pointer advances

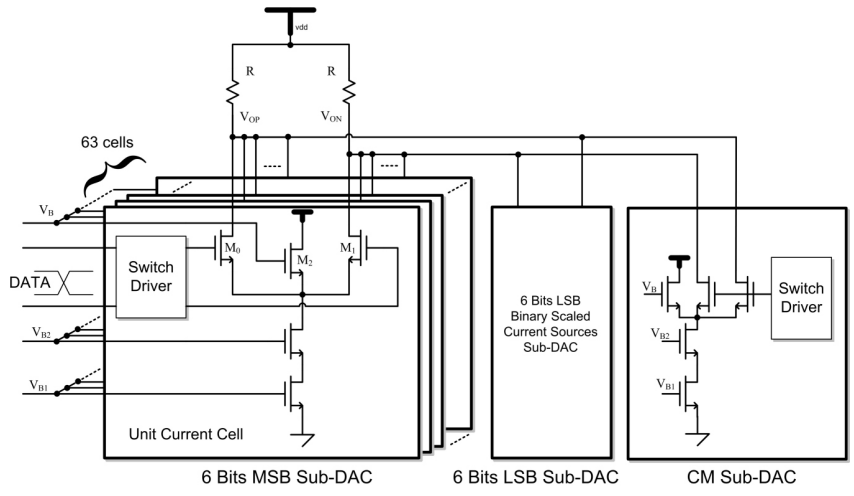

Fig. 5. The architecture of the analog current steering DAC

with the magnitude of the activated subDAC. Notice that subDAC can be negative, positive or zero. If its value is zero, the pointer does not change.

The outputs of the circular MSB unit controller not only identify the active unit cells but also determine the sign of their output with respect to the related subDAC value. The unit element, as will be described shortly, can generate bipolar signals. It is controlled by 2 input signals in a 2 's complement fashion.

\section{Circuit Implementation}

The digital section of the DAC and signal processing algorithm is designed and synthesized using Verilog-HDL in a 90nm CMOS technology. The cell count of the digital section is 1042 . The area occupied by the digital section is negligible compared to the active area of the analog current source section. The architecture of the analog current steering DAC section is shown in Fig. 5.

The unit elements within the 6-bits MSB subDAC section should be able to generate bipolar signals including zero output. This problem is solved using the structure shown in Fig. 5. Each unit cell receives 2 control inputs from the digital section. The data is sent in 2's complement form. If the input is $1(01)$ or $-1(10)$, the reference current is routed towards the appropriate output terminal whereas if the input is $0(00)$, the reference current goes to the supply. This way, the cell can generate bipolar output signal in a differential fashion.

The architecture of the unit cell within the LSB subDAC is exactly identical to the MSB subDAC. The reference current levels of the former are binary scaled to reduce required silicon area. The switch drivers, present within both subDAC, reduce the signal swing of the control signals to reduce the disturbance on the cell cascaded current reference as well as improve the switching speed of the cell.

The simplified internal structure $\mathrm{CM}$ subDAC is shown in Fig. 5. The reference currents of the CM subDAC is binary weighted to save space. It is obvious that scaling the reference currents of the CM subDAC aggravates the linearity of this subDAC. This is not an issue because as it is clear from Fig. 3, the output common mode signal is de-correlated from the input signal by the proposed algorithm so that the errors introduced 

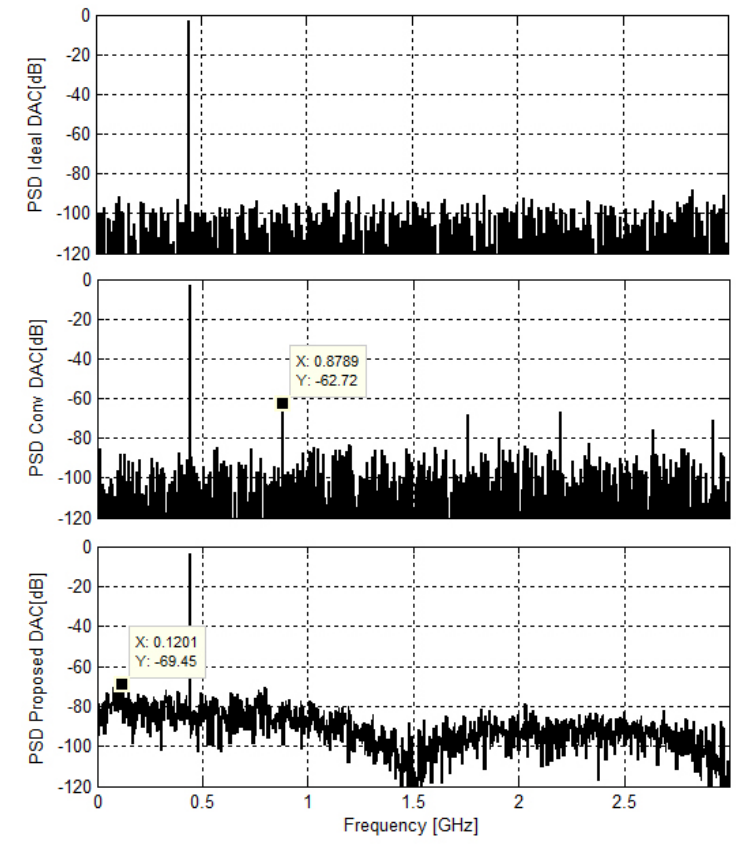

Fig. 6. The performance comparison of an ideal DAC, a conventional DAC and the proposed DAC

by the CM subDAC degrade only the signal to noise ratio of the whole system and not its linearity.

The key blocks of the proposed architecture have been simulated at the transistor level using a $90 \mathrm{~nm}$ CMOS technology. The simulation results achieve a speed of $6 \mathrm{GHz}$ with the expected signal accuracy. The system converts the output current into voltage with $50 \Omega$ output resistors. The expected total current consumption is $13 \mathrm{~mA}$ with a peak-topeak differential output of $500 \mathrm{mV}$.

\section{MisMATCH ERROR}

The accuracy of the proposed scheme depends on the accuracy and the matching of the time-interleaved DACs and the accuracy of the fast residual and LSB DAC. The accuracy of the slow DACs is ensured, in addition to a careful layout and signal control distribution by the lower speed and the return to zero. The switching speed of individual cell within MSB DAC is 6 times smaller than the actual sampling clock. The unity element sharing, described shortly, enhances the matching between the slow DACs obtaining a sort of dynamic element matching of errors.

The accuracy required for the fast converter is relaxed because its full scale is more than an order of magnitude of the full scale of the entire converter. The key limit here is to obtain the matching between the full scale amplitudes of the two converters. A possible solution is to use foreground calibration to match the output of the 5 LSB of the slow DAC with the full scale of the LSB [4].

\section{Simulation Results}

The Fig. 6 shows the comparison of the power spectral densities of an Ideal DAC whose unit elements are perfectly matched, a Conventional DAC array of non ideal unit elements and the proposed DAC with again non ideal unit elements. All of the current references shown in Fig. 1 are mismatched with a mismatch term that is normal distributed with a standard deviation \pm 3 LSB.

As it is clear from the figure, proposed scheme trades the signal to noise ratio performance of the DAC to its spurious free dynamic range, as one would expect from a dynamic element matching scheme. The SFDR improves around $7 \mathrm{~dB}$ (from $-62 \mathrm{~dB}$ to $-69 \mathrm{~dB}$ ) once the scheme is enabled. The signal to noise ratio (SNR) on the other hand degrades approximately $9 \mathrm{~dB}$ (from $-71 \mathrm{~dB}$ to $-62 \mathrm{~dB}$ ) calculated within the signal bandwidth that is half of the DAC Nyquist bandwidth. Note that the simulation results do not incorporate the SNR degradation due to the clock jitter.

This improvement is obtained without actually increasing the size of the analog section of the DAC but by only digital signal processing and exploiting the intrinsic oversampling requirement of the system. Furthermore, the proposed architecture allows to reduce the switching speed to $1 / 6^{\text {th }}$ of the equivalent sampling frequency.

\section{CONCLUSIONS}

A 12-bit multi-rate segmented time-interleaved current steering DAC with unity-elements sharing is presented. The DAC is segmented to MSB and LSB sections. Proposed novel algorithm controlling the MSB segment of the high speed DAC improves the spurious free dynamic range of the DAC $7 \mathrm{~dB}$ at $1 / 13^{\text {th }}$ of a $6 \mathrm{GHz}$ sampling clock frequency. The architecture utilizes time-interleaved and return-to-zero techniques to reduce the duty cycle of individual current cell within the MSB DAC array and to improve the linearity of the system. The MSB cell array is activated in circular fashion to implement data weighted averaging. The algorithm trades effectively the signal to noise ratio of the system with its spurious performance. LSB segment of the DAC operating at full speed is implemented using simple binary weighted cells to save space. A common mode correction DAC is also incorporated to the system to reduce common mode variation at the differential output terminals. The DAC is implemented in a 90nm digital CMOS technology and consumes $13 \mathrm{~mA}$ from a single $1.5 \mathrm{~V}$ supply.

\section{REFERENCES}

[1] Z. Zhou and G. L. Rue, "A 12-bit nonlinear dac for direct digital frequency synthesis," IEEE Trans. Circuits Syst. I, vol. 55, no. 9, pp. 2459-2468, Oct. 2008.

[2] R. T. Baird and T. S. Fiez, "Linearity enhancement of multibit $\Delta \Sigma$ A/D and D/A converters using data weighted averaging," IEEE Trans. Circuits Syst. II, vol. 42, no. 12, pp. 753-762, Dec. 1995.

[3] L. R. Carley, "A noise-shaping coder topology for 15+ bit converters," IEEE J. Solid-State Circuits, vol. 24, no. 2, pp. 267-273, Apr. 1989.

[4] M. Mitsuishi, H. Yoshida, M. Sugawara, Y. Kunisaki, S. Nakamura, S. Nakaigawa, and H. Suzuki, "A sub-binary-weighted current calibration technique for a 2.5v 100ms/s 8bit adc," Proceedings of the 24th European Solid-State Circuits Conference(ESSCIRC'98), pp. 420-423, Sept. 1998. 